\title{
Risk factors for atelectasis of the middle lobe after right upper lobectomy: preoperative bronchial diameter and stapling of the fissure
}

\author{
Yasushi Mizukami^, Yuki Takahashi, Ryunosuke Maki, Hirofumi Adachi \\ Department of Thoracic Surgery, National Hospital Organization, Hokkaido Cancer Center, Hokkaido, Japan \\ Contributions: (I) Conception and design: Y Mizukami; (II) Administrative support: Y Mizukami; (III) Provision of study materials or patients: Y \\ Mizukami, H Adachi; (IV) Collection and assembly of data: Y Mizukami, H Adachi; (V) Data analysis and interpretation: All authors; (VI) Manuscript \\ writing: All authors; (VII) Final approval of manuscript: All authors. \\ Correspondence to: Yasushi Mizukami. Department of Thoracic Surgery, National Hospital Organization, Hokkaido Cancer Center, 2-3-54 Kikusui 4-jo, \\ Shiroishi-ku, Sapporo-shi, Hokkaido 003-0804, Japan. Email: mizukam@gmail.com.
}

Background: Atelectasis of the middle lobe after right upper lobectomy is often seen. However, the risk
factors for atelectasis are uncertain. Therefore, we assessed cases in our institution and investigated risk
factors for atelectasis of the middle lobe following right upper lobectomy.
Methods: We identified 354 cases in which right upper lobectomy had been performed in our institution
between January 2009 and December 2018, and 342 were included in this retrospective analysis. We divided
patients into two groups according to the presence of postoperative atelectasis of the middle lobe, and
then preoperative clinical variables and perioperative variables were compared between the two groups.
Multivariable analyses for postoperative atelectasis of the middle lobe were performed using the logistic
regression model. Results: Middle lobe atelectasis was detected in 59 cases (17.3\%). Multivariable analysis demonstrated that the preoperative diameter of the middle lobe bronchus $[\mathrm{P}=0.012$; confidence interval (CI), 0.525-0.930] and stapling of the fissure between the upper and middle lobes ( $\mathrm{P}=0.004$; CI, 1.997-37.050) were independent risk factors for postoperative atelectasis of the middle lobe.

Conclusions: A small preoperative diameter of the middle lobe bronchus and stapling of the fissure between the upper and middle lobes are risk factors for middle lobe atelectasis following right upper lobectomy.

Keywords: Lung; pulmonary resection; right upper lobectomy; atelectasis of the middle lobe

Submitted Jul 01, 2021. Accepted for publication Aug 26, 2021.

doi: $10.21037 /$ jtd-21-1081

View this article at: https://dx.doi.org/10.21037/jtd-21-1081

\section{Introduction}

Atelectasis of the middle lobe after right upper lobectomy is often seen, and the rates are higher than for other lobes $(1,2)$. Atelectasis can result in pneumonia or respiratory dysfunction. Some studies have reported that chronic obstructive pulmonary disease or a short distance from the carina to the middle lobe orifice could be risk factors for middle lobe atelectasis $(2,3)$. However, the risk factors are uncertain, and the condition cannot be prevented. Torsion of the middle lobe is rarely seen after right upper lobectomy, but it is a serious complication $(4,5)$. Torsion of the lobe

\footnotetext{
^ ORCID: 0000-0002-2099-1687.
} 


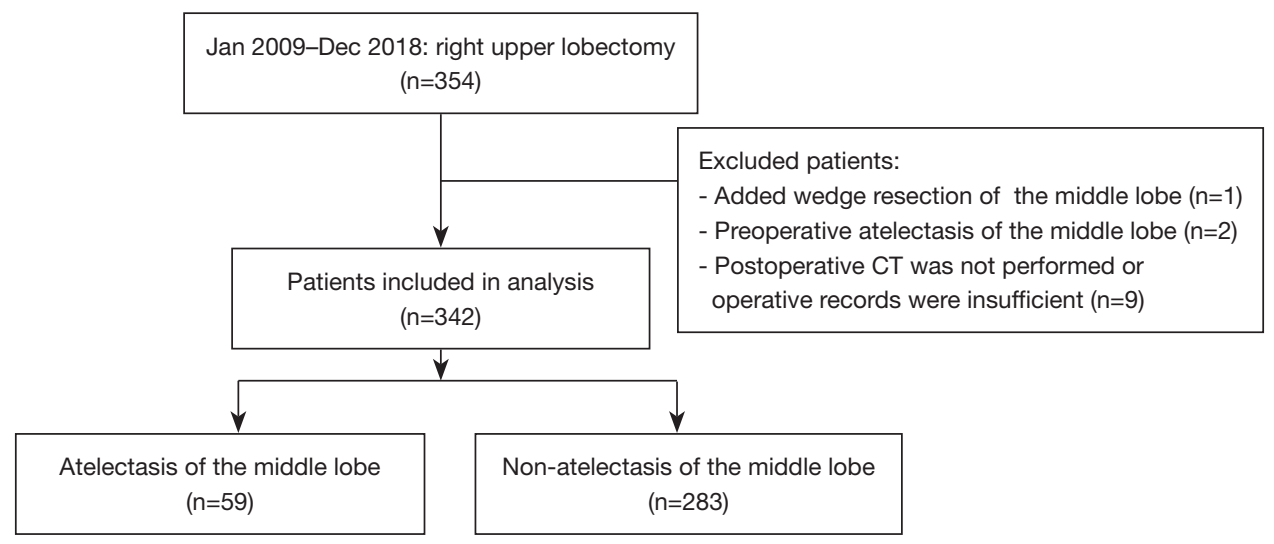

Figure 1 Flow chart of the selection of patients analyzed in this study. CT, computed tomography.

occurs in $0.089-0.3 \%$ of patients following lobectomy $(6,7)$. Anatomical rearrangement of bronchi by expansion of the lower lobe after upper lobectomy including kinking of bronchi may be related to atelectasis of the middle lobe (2). We hypothesized that an incomplete and dense fissure for stapling of the fissure between the upper and middle lobes may deform the middle lobe and may introduce kinking of bronchi more frequently than a complete fissure without stapling. Therefore, we assessed cases in our institution and investigated risk factors for atelectasis of the middle lobe following right upper lobectomy to test our hypothesis.

We present the following article in accordance with the STROBE reporting checklist (available at https://dx.doi. org/10.21037/jtd-21-1081).

\section{Methods}

This study retrospectively reviewed the records of patients who underwent right upper lobectomy at a single center, Hokkaido Cancer Center in Sapporo, Japan, between January 1, 2009 and December 31, 2018. The study was conducted in accordance with the Declaration of Helsinki (as revised in 2013). The ethics approval for this study was granted by the ethics committee of Hokkaido Cancer Center (approval number: 31-22); the requirement to obtain informed consent directly was waived and informed consent was obtained in the form of opt-out on the website. We identified 354 cases in which right upper lobectomy had been performed, and 342 were included in the analysis (Figure 1).

All cases were performed by 5 surgeons trained for 7 years or longer. The operative procedure was performed in the lateral decubitus position under general anesthesia.
Although epidural anesthesia was performed in nearly all cases, in some cases, epidural anesthesia was not performed because the patient was taking antithrombotic drugs, the patient had a deformity of thoracic vertebrae or a history of vertebrae operation, request of the patient, difficulty with insertion of epidural tube, etc. The trachea was canalized with a double-lumen tube for selective ventilation of the lung. A $4-\mathrm{cm}$ minithoracotomy was made in front of the anterior line of the latissimus dorsi muscle on the posterolateral incision line (basically within the fourth or fifth intercostal space), and a 5-mm port for the thoracoscope was inserted at the mid-axillary line (in the fifth or sixth intercostal space, at a point that looks down the major fissure). A 2-cm assistant working port was inserted at a posterior location on the same line (in the sixth or seventh intercostal space). A protective film and ring device (LAPPROTECTOR ${ }^{\mathrm{TM}}$; Hakko Co. Ltd., Tokyo, Japan) for protecting the wounds of the operator's port and assistant working port were used. Thoracoscopic surgery was converted to open thoracotomy if necessary, but this was rare. When necessary, open thoracotomy was performed through the posterolateral incision. If adhesions and incomplete lobulation were not identified intraoperatively, the ascending A2 was divided, and then the interlobar fissure was divided by staplers. The staple line was placed on the interlobar fissure unless the interlobar fissure was close to the tumor. Subsequently, the pulmonary vein and superior trunk (and A3) were divided. Staple suturing of the bronchial stump was performed by applying cartilage longitudinally onto the mucosa, according to Sweet's technique. If incomplete lobulation was identified, the pulmonary vein, artery, and bronchus were divided, and then the interlobar fissure was divided by staplers. Finally, 

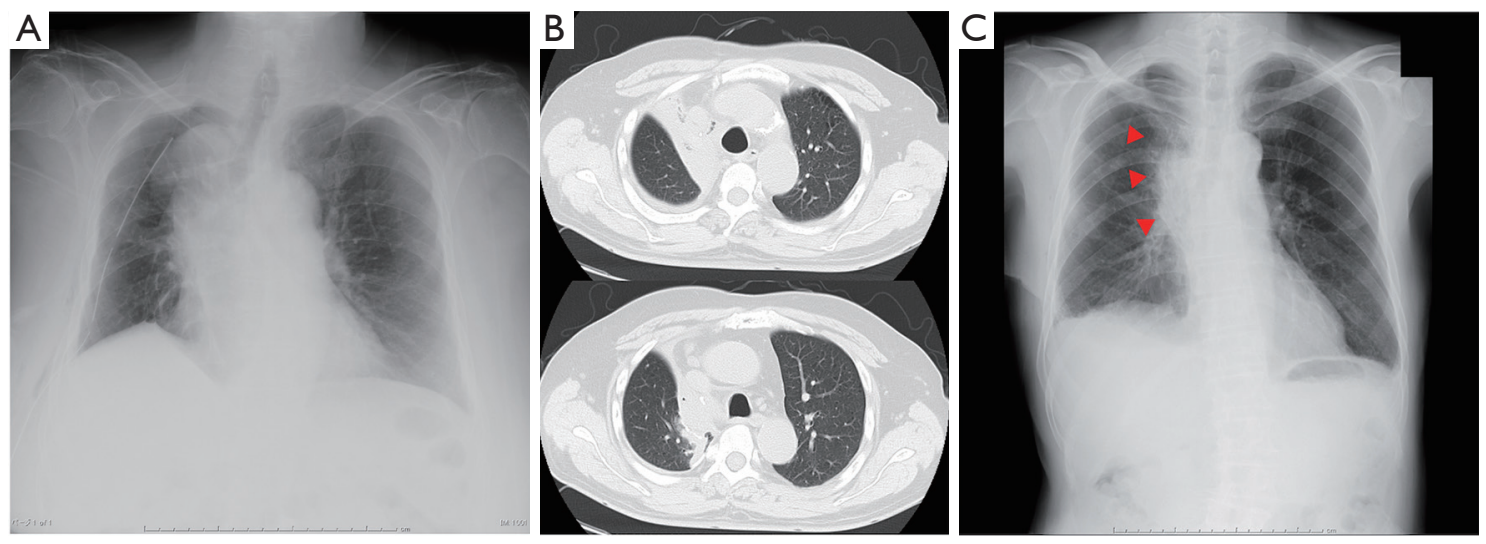

Figure 2 Schema of atelectasis of the middle lobe after right upper lobectomy. Representative X-ray (A) or computed tomography (B) images used to evaluate middle lobe atelectasis. Partial atelectasis on X-ray (C) indicate a weak mediastinal shift and opacity including gas (arrowhead).

lymphadenectomy was performed as necessary. Fixation of the middle lobe to the remaining lower lobe to prevent pulmonary torsion was not performed. For postoperative analgesia, oral administration of the nonsteroidal antiinflammatory drug celecoxib or acetaminophen was started on postoperative day 1 . The patients received mucolytic agents routinely. When sputum retention was not improved by physiotherapy regardless of middle lobe atelectasis, mini-tracheostomy (Mini-Trach II Seldinger Kit ${ }^{\mathrm{TM}}$, Smiths Medical ASD, Inc., Minneapolis, USA) was performed.

We investigated all patients in whom atelectasis of the middle lobe was detected on chest X-ray or computed tomography (CT) during follow-up regardless of the presence/absence of symptoms (Figure 2). Chest X-ray was mainly performed perioperative period during admission. CT was performed during follow-up duration after discharge unless pneumonia was doubted. We excluded cases in which a localized shadow along the staple line or continuous hemoptysis was seen. Basically, chest X-ray was performed immediately after surgery and on postoperative days 1, 2, 4, and 7. CT was performed when pneumonia was suspected or for regular follow-up after surgery every 3 to 6 months. Pre- and perioperative data were collected retrospectively from the hospital's electronic medical records. Chronic obstructive pulmonary disease was diagnosed using the ratio of forced expiratory volume in one second to forced vital capacity $<70 \%$ on spirometry after bronchodilator administration. The pulmonary fissure was classified intraoperatively into four grades according to Craig et al. (8). The pre- and postoperative diameters of the middle lobe bronchus were measured on $1.25-\mathrm{mm}$ axial CT slices by senior thoracic surgeons as shown in Figure $3 A, 3 B$. The maximum diameter at $5 \mathrm{~mm}$ from the orifice of the middle lobe bronchus was used. Postoperative $\mathrm{CT}$ within 6 months was used. The distance from the carina to the middle lobe orifice was measured on a coronal CT view (Figure 3C,3D).

We divided the patients into two groups (atelectasis group and non-atelectasis group) according to the presence of postoperative atelectasis of the middle lobe, and then preoperative clinical variables and perioperative variables were compared between the two groups.

\section{Statistical analysis}

Continuous variables are summarized as the mean (standard deviation). All statistical analyses were performed using $\mathrm{JMP}^{\circledR} 13$ (SAS Institute Inc., Cary, NC, USA). Pearson's chi-square test and Student's $t$-test were used for statistical analyses. For exploratory analyses, logistic regression models were prepared to determine the association between clinical characteristics and postoperative atelectasis of the middle lobe. Selection of a priori variables was based on previous literature and included chronic obstructive pulmonary disease and distance from the carina to the middle lobe orifice. The final multivariable model included those variables for which the statistical significance was $\mathrm{P} \leq 0.10$ in univariate analysis. Values of $\mathrm{P}<0.05$ were 

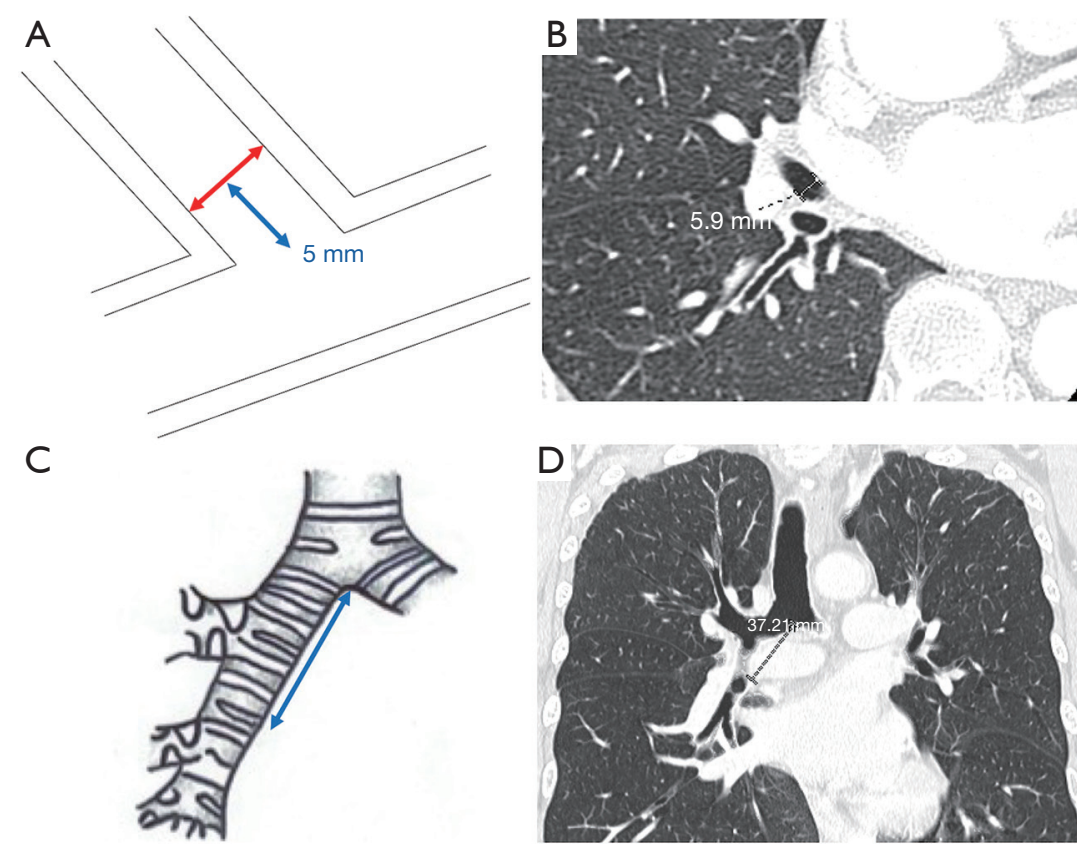

Figure 3 Method to measure the diameter of the middle lobe bronchus. The internal diameter of the middle lobe bronchus was measured from axial images (red double-headed arrow). The maximum diameter was used at $5 \mathrm{~mm}$ from the middle lobe orifice on the slice which the middle lobe bronchus was the thickest. A schematic image (A) and a representative computed tomography image (B) are shown. Method to measure the distance from the carina to the middle lobe orifice (blue double-headed arrow) (C,D).

considered significant.

\section{Results}

Middle lobe atelectasis was detected in 59 cases (17.3\%). 17 cases were conversion to open thoracotomy due to strong adhesions (15 cases) and bleeding ( 2 cases). Patient characteristics are listed in Table 1. Univariate analyses were performed. The preoperative diameter of the middle lobe bronchus $(\mathrm{P}=0.047)$ was significantly smaller, and the fissure grade $(\mathrm{P}<0.0001)$ was significantly higher in the atelectasis group than in the non-atelectasis group. The rate of stapling of the fissure between the upper and middle lobes $(\mathrm{P}=0.0002)$ was significantly higher, and the postoperative diameter of the middle bronchus $(\mathrm{P}<0.0001)$ was significantly smaller in the atelectasis group than in the non-atelectasis group. Detection of middle lobe atelectasis was 1 day postoperatively (median, inter-quartile range, $0.75-2$ ). Bronchoscopy was not performed for sputum suction. However, mini-tracheostomy was performed in five cases in the atelectasis group. In 54 of 59 patients $(91.5 \%)$, atelectasis improved over time following the mini- tracheostomy, medical rehabilitation, or phlegm expulsion assistance. In 57 of 59 cases, the atelectasis was diagnosed with chest X-ray. In 13 of 59 cases, atelectasis was present at the time of postoperative bronchial diameter measurement. The median of the period until the atelectasis disappeared was 24 days (range, 1-1,088 days). Diagnoses showed a high proportion of lung cancer between the two groups. There were few diseases other than lung cancer, with 2 cases of metastasis in the atelectasis group, and 12 cases of metastasis and 3 cases of benign nodules in the non-atelectasis group.

In univariate analysis, the $\mathrm{P}$ value was $\leq 0.10$ for stapling of the fissure between the upper and middle lobes, fissure grade, and fissureless technique. However, these three variables act as covariates because they are all related. Therefore, only stapling of the fissure between the upper and middle lobes was adapted in multivariable logistic regression models. Multivariable analysis demonstrated that operative time $(\mathrm{P}=0.04)$, the preoperative diameter of the middle lobe bronchus $(\mathrm{P}=0.012)$, and stapling of the fissure between the upper and middle lobes $(\mathrm{P}=0.004)$ were independent risk factors for postoperative atelectasis of the middle lobe (Figure 4). 
Table 1 Univariate analysis of patient characteristics

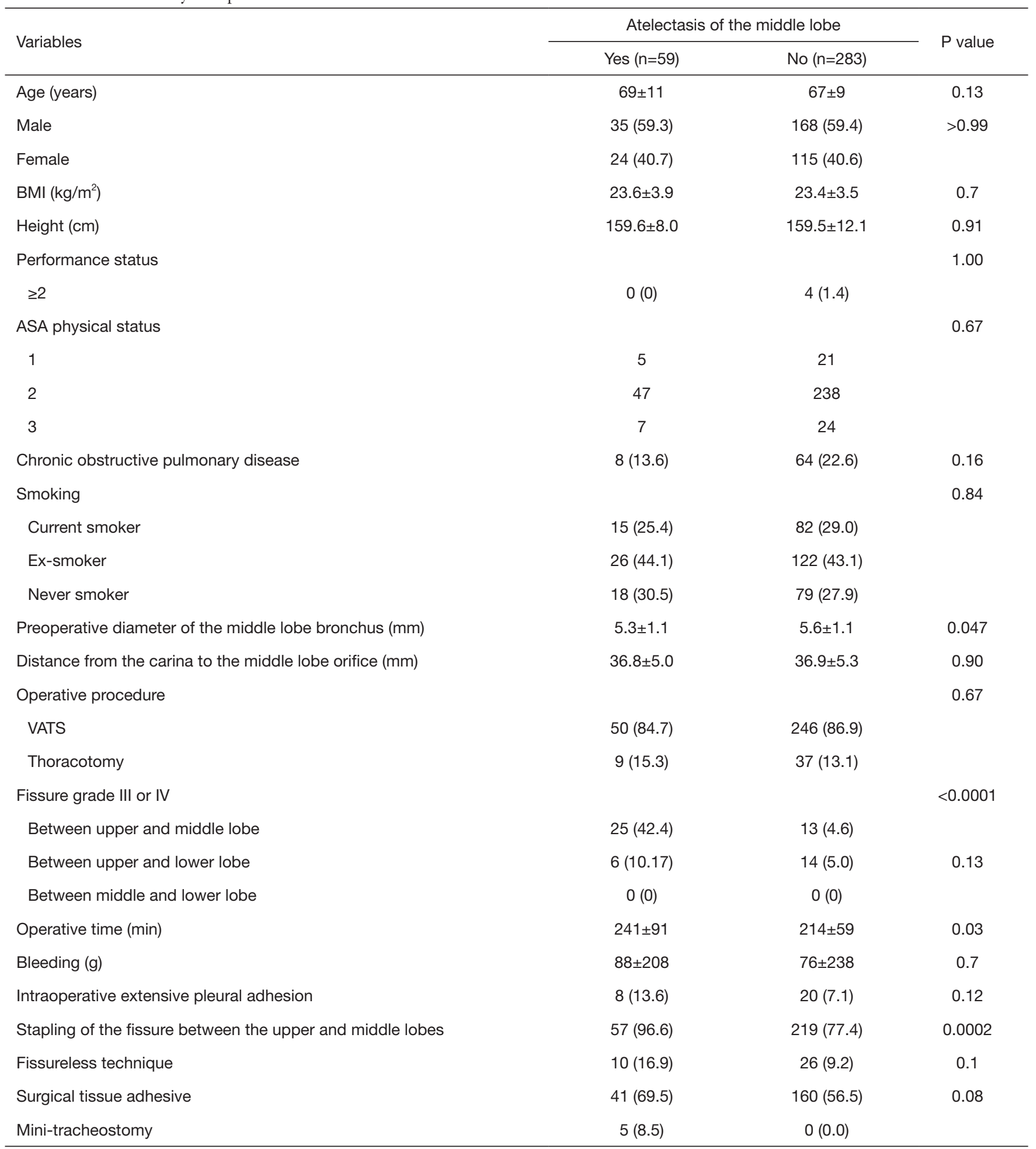

Table 1 (continued) 
Table 1 (continued)

\begin{tabular}{|c|c|c|c|}
\hline Variables & \multicolumn{2}{|c|}{ Atelectasis of the middle lobe } & $P$ value \\
\hline Diagnosis & & & 0.75 \\
\hline Lung cancer & $57(16.7)$ & $268(78.4)$ & \\
\hline Other & $2(0.6)$ & $15(4.4)$ & \\
\hline Chest drainage duration & $3.9 \pm 2.9$ & $4.1 \pm 6.4$ & 0.62 \\
\hline Duration from operative day to first postoperative CT (days) & $114(80.0)$ & $126(167.0)$ & 0.40 \\
\hline Postoperative diameter of the middle bronchus (mm) & $3.1 \pm 1.9$ & $4.4 \pm 1.4$ & $<0.0001$ \\
\hline Follow-up duration (days) & $1,750 \pm 903$ & $1,860 \pm 966$ & 0.40 \\
\hline
\end{tabular}

Values represent $\mathrm{n}(\%)$, mean \pm SD. BMI, body mass index; VATS, video-assisted thoracoscopic surgery; ASA, American Society of Anesthesiologists.

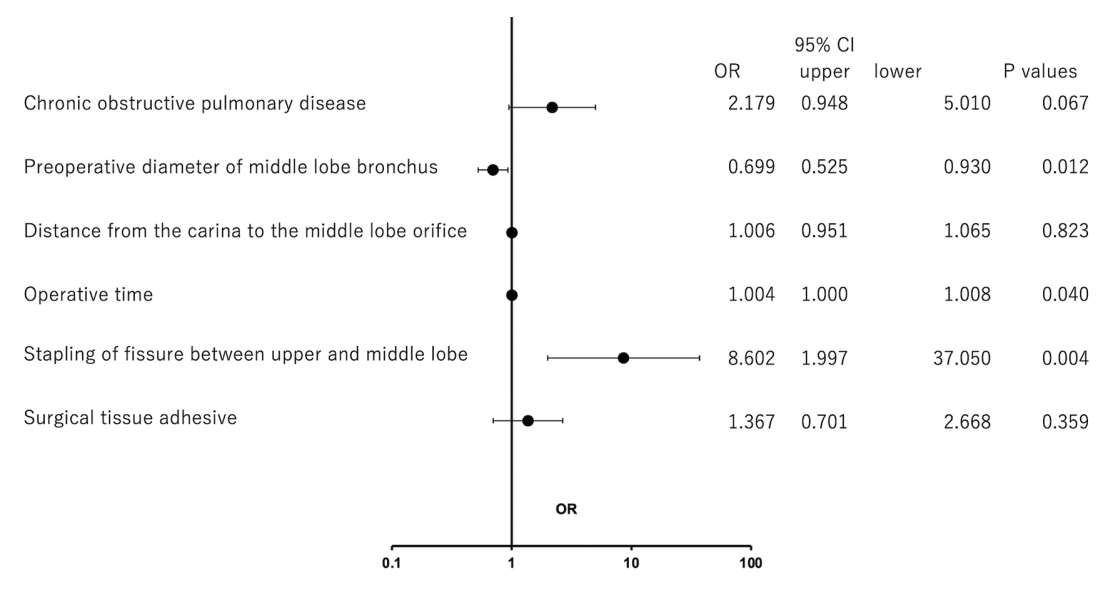

Figure 4 Exploratory logistic regression models. Forest plot of odds ratios for the six possible risk factors. Forest plot of the odds ratios, indicated by circles on the $\mathrm{x}$-axis, for postoperative atelectasis of the middle lobe after right upper lobectomy. Horizontal lines show the $95 \%$ confidence interval. CI, confidence interval; OR, odds ratio.

\section{Discussion}

Atelectasis of the middle lobe after right upper lobectomy is often seen, and the frequency is higher than with the other lobes $(1,2)$. Atelectasis can result in pneumonia or respiratory dysfunction. However, the risk factors for this condition are uncertain. Here, we identified three risk factors for atelectasis of the middle lobe after upper lobectomy.

The rate of middle lobe atelectasis following right upper lobectomy is $11.4-12 \%(2,3,9)$. The diagnoses of atelectasis in these studies were made with various methods including bronchoscopy, X-ray, or CT. In our study, the rate of middle lobe atelectasis following right upper lobectomy was $17.3 \%$, which was slightly higher than in these previous reports. We studied atelectasis identified on imaging including CT regardless of bronchoscopy, and this may explain these differences in the rate of middle lobe atelectasis. In addition, we experienced no cases requiring bronchoscopy.

Right upper lobectomy is followed by a tendency to develop atelectasis of the middle lobe $(1,10)$. Anatomical rearrangement of bronchi due to expansion of the lower lobe after upper lobectomy including kinking of the 
bronchus may be related to atelectasis of the middle lobe (2). However, the reason is unclear. We also believe that the cause of atelectasis of the middle lobe after upper lobectomy is kinking of the middle lobe bronchus. Matsuda et al. (3) reported that a short distance from the carina to the middle lobe orifice was the only risk factor. If so, we thought that patients with a smaller body size who underwent right upper lobectomy may develop atelectasis of the middle lobe more frequently. However, this was not necessarily so, and our results indicated that the distance from the carina to the middle lobe orifice was not significantly different between the two groups. We presume that a smaller postoperative size of the middle lobe bronchus can be influenced by kinking of the bronchus to some extent. More importantly, we suggest that an incomplete and dense fissure for stapling of the fissure between the upper and middle lobes may deform the middle lobe and introduce kinking of the bronchus more frequently than a complete fissure without stapling. Our results support this hypothesis. We have rarely experienced the middle lobe atelectasis after lower lobectomy. It is assumed the reason is because the rate of incomplete fissure between the middle lobe and the lower lobe is lower than it between the upper lobe and the middle lobe or middle lobe bronchus is tend to be straight line to the intermediate bronchus after lower lobectomy.

Fissureless technique is the way which the dense interlobar fissure is finally divided by staplers. It can decrease postoperative prolonged air leakage. We had predicted that fissureless technique was concerned with atelectasis. However, our results indicated that although the cases of atelectasis using fissureless technique were tend to be higher than non-atelectasis group, the differences between them was insignificant. Considering our results, fissureless technique might have been tend to cause stapling on the upper lobe side because the fissure is stapled finally. The operative time was slightly longer in the atelectasis group than the non-atelectasis group. We thought that the reason was that a little more time was required to divide the interlobar fissure by stapling due to an incomplete or dense fissure. However, it is assumed that the odds ratio is just about 1 and ignorable clinically.

Most of all cases of atelectasis improved within one month and disappearance of postoperative bronchial edema may associate with improvement of the atelectasis. On the other hand, two rare cases of improvement of long term atelectasis for about 3 years was detected on followCT. These cases were not atelectasis in perioperative term and the atelectasis was detected during follow-up period.
Both cases were stapled the fissure between the upper and middle lobes and maintained postoperative small bronchial diameter. Thus, it is presumed that the atelectasis prone to develop even with a small mucus plug.

Approximately $30 \%$ of thoracic surgeons who answered a questionnaire had seen one or more cases of pulmonary torsion, and $57 \%$ routinely fix the middle lobe to the remaining lobe after right upper or lower lobectomy (11). However, torsion of the lobe is rare and occurs in 0.089 $0.3 \%$ of patients following lobectomy $(6,7)$. In our study, pulmonary torsion was not seen, although fixation of the middle lobe to the remaining lower lobe to prevent pulmonary torsion was not performed. We observed that the remaining lobes were inflated in the natural position. Thus, we believe that inflated lobes are fixed in the thoracic cavity and are the reason that pulmonary torsion was not seen. Sugita et al. (12) reported that long-term respiratory function after right upper lobectomy with video-assisted thoracoscopic surgery may be significantly better than that via thoracotomy. We have not investigated the long-term respiratory function. However, we think that middle lobe atelectasis after right upper lobectomy does not affect longterm respiratory function because most cases of atelectasis improved.

Mi et al. (13) reported that the mean diameter of the middle bronchus is $12.2 \mathrm{~mm}$, which was wider than in our study. The reason may be that the heights of our patients are less than those in their report, or that different methods were used to measure each population. On the other hand, the present study indicates that a small bronchus size is associated with postoperative atelectasis of the middle lobe. This idea should be evaluated further in other institutions and countries.

The present study has some limitations that need to be considered when interpreting the results. First, this investigation used a single-institution retrospective design. A prospective or multicenter study is required to further evaluate the prediction of postoperative atelectasis of the middle lobe after right upper lobectomy. Second, our method for measurement of the bronchus was on an axial or coronal CT view, and the use of measurements such as three-dimensional CT may achieve a more accurate result for the assessment of the bronchus. Third, although no significant difference was seen between the two groups in terms of video-assisted thoracoscopic surgery or thoracotomy, we could not assess postoperative pain, which may be associated with postoperative atelectasis of the middle lobe after right upper lobectomy. Including these 
points, unexpected risk factors for postoperative atelectasis of the middle lobe after right upper lobectomy may be identified in the future.

\section{Conclusions}

A small preoperative diameter of the middle lobe bronchus and stapling of the fissure between the upper and middle lobes are risk factors for middle lobe atelectasis following right upper lobectomy. Further prospective studies of this method for predicting the middle lobe atelectasis after right upper lobectomy should be conducted.

\section{Acknowledgments}

The authors would like to acknowledge all the staff involved in the care of the patients in the Department of Thoracic Surgery at the National Hospital Organization, Hokkaido Cancer Center.

Funding: None.

\section{Footnote}

Reporting Checklist: The authors have completed the STROBE reporting checklist. Available at https://dx.doi. org/10.21037/jtd-21-1081

Data Sharing Statement: Available at https://dx.doi. org/10.21037/jtd-21-1081

Peer Review File: Available at https://dx.doi.org/10.21037/ jtd-21-1081

Conflicts of Interest: All authors have completed the ICMJE uniform disclosure form (available at https://dx.doi. org/10.21037/jtd-21-1081). The authors have no conflicts of interest to declare.

Ethical Statement: The authors are accountable for all aspects of the work in ensuring that questions related to the accuracy or integrity of any part of the work are appropriately investigated and resolved. The study was conducted in accordance with the Declaration of Helsinki (as revised in 2013). The ethics approval for this study was granted by the ethics committee of Hokkaido Cancer Center (approval number: 31-22); the requirement to obtain informed consent directly was waived and informed consent was obtained in the form of opt-out on the website.
Open Access Statement: This is an Open Access article distributed in accordance with the Creative Commons Attribution-NonCommercial-NoDerivs 4.0 International License (CC BY-NC-ND 4.0), which permits the noncommercial replication and distribution of the article with the strict proviso that no changes or edits are made and the original work is properly cited (including links to both the formal publication through the relevant DOI and the license). See: https://creativecommons.org/licenses/by-nc-nd/4.0/.

\section{References}

1. Korst RJ, Humphrey CB. Complete lobar collapse following pulmonary lobectomy. Its incidence, predisposing factors, and clinical ramifications. Chest 1997;111:1285-9.

2. Stolz AJ, Schutzner J, Lischke R, et al. Predictors of atelectasis after pulmonary lobectomy. Surg Today 2008;38:987-92.

3. Masuda Y, Marutsuka T, Suzuki M. A risk factor for kinked middle lobar bronchus following right upper lobectomy. Asian Cardiovasc Thorac Ann 2014;22:955-9.

4. Kanemitsu S, Tanaka K, Suzuki H, et al. Pulmonary torsion following right upper lobectomy. Ann Thorac Cardiovasc Surg 2006;12:417-9.

5. Childs L, Ellis S, Francies O. Pulmonary lobar torsion: a rare complication following pulmonary resection, but one not to miss. BJR Case Rep 2017;3:20160010.

6. Cable DG, Deschamps C, Allen MS, et al. Lobar torsion after pulmonary resection: presentation and outcome. J Thorac Cardiovasc Surg 2001;122:1091-3.

7. Keagy BA, Lores ME, Starek PJ, et al. Elective pulmonary lobectomy: factors associated with morbidity and operative mortality. Ann Thorac Surg 1985;40:349-52.

8. Craig SR, Walker WS. A proposed anatomical classification of the pulmonary fissures. J R Coll Surg Edinb 1997;42:233-4.

9. Kim DH, Moon DH, Kim HR, et al. Effect of inferior pulmonary ligament division on residual lung volume and function after a right upper lobectomy. Interact Cardiovasc Thorac Surg 2019;28:760-6.

10. Uzieblo M, Welsh R, Pursel SE, et al. Incidence and significance of lobar atelectasis in thoracic surgical patients. Am Surg 2000;66:476-80.

11. Wong PS, Goldstraw P. Pulmonary torsion: a questionnaire survey and a survey of the literature. Ann Thorac Surg 1992;54:286-8.

12. Sugita Y, Kuroda H, Sakata S, et al. How preserved 
regional pulmonary function after thoracoscopic segmentectomy in clinical stage I non-small cell lung cancers in right upper lobe. Gen Thorac Cardiovasc Surg 2021;69:960-6.

Cite this article as: Mizukami Y, Takahashi Y, Maki R, Adachi $\mathrm{H}$. Risk factors for atelectasis of the middle lobe after right upper lobectomy: preoperative bronchial diameter and stapling of the fissure. J Thorac Dis 2021;13(10):5649-5657. doi: 10.21037/jtd-21-1081
13. Mi W, Zhang $\mathrm{C}$, Wang $\mathrm{H}$, et al. Measurement and analysis of the tracheobronchial tree in Chinese population using computed tomography. PLoS One 2015;10:e0123177. 